\title{
SILAR-Based Application of Various Nanopillars on GaN-Based LED to Enhance Light-Extraction Efficiency
}

\author{
S. C. Shei \\ Department of Electrical Engineering, National University of Tainan, Tainan 700, Taiwan \\ Correspondence should be addressed to S. C. Shei; scshei@mail.nutn.edu.tw
}

Received 31 August 2013; Accepted 5 October 2013

Academic Editor: Liang-Wen Ji

Copyright ( 92013 S. C. Shei. This is an open access article distributed under the Creative Commons Attribution License, which permits unrestricted use, distribution, and reproduction in any medium, provided the original work is properly cited.

\begin{abstract}
We reported the various nanopillars on GaN-based LED to enhance light-extraction efficiency prepared by successive ionic layer adsorption and reaction method (SILAR). Indium tin oxide (ITO) with thickness of $1 \mu \mathrm{m}$ as transparent contact layer was grown to improve the electrical characteristics of the LEDs, including series resistance and operating voltage. SILAR-deposition $\mathrm{ZnO}$ nanoparticles on $\mathrm{SiO}_{2}$ were used as etching nanomasks. Multiple nanopillars were simultaneously formed on overall surfaces of ITO $\mathrm{p}$ - and $\mathrm{n}-\mathrm{GaN}$ by ICP etching. The proposed GaN-based LEDs with nanopillars increase light output power by $7 \%-20.3 \%$ (at $20 \mathrm{~mA}$ ) over that of regular GaN-based LEDs. The difference in light output power can be attributed to differences in materials and shapes of nanopillars, resulting in a reduction in Fresnel reflection by the roughened surface of GaN-based LEDs.
\end{abstract}

\section{Introduction}

GaN-based materials and their related ternary compounds such as $\mathrm{AlGaN}$ and InGaN have attracted much attention. In the past decades, high-brightness GaN-based LEDs have penetrated the markets of displays, traffic signals, and even solid-state lighting [1]. It is required to further extend the application arm of GaN-based LED to projectors automobile headlight and even general lighting and further improvement on optical power and light-extraction efficiency are eagerly required. Several methods, including surface roughening techniques [2-5], inclined sidewall etching [6], patterned sapphire substrates $[7,8]$, and the incorporation of highly reflective omnidirectional reflectors (ODRs) [9], have been shown to effectively improve the light-extraction efficiency. Among these methods, surface roughening is one of the most efficient to provide an enhancement factor for the extraction efficiency due to increased random scattering events that occur at the roughened surfaces. However, the etching techniques have inherent limit to the thickness of $\mathrm{p}$ GaN layer, which is $\sim 300 \mathrm{~nm}$ [10-12]. Therefore, the etching process must be precisely controlled to avoid deterioration of electrical properties. Moreover, although other approaches involving the synthesis of $\mathrm{ZnO}$ nanorods and the spin coating of polystyrene nanospheres have been developed for GaNbased LEDs, separate process steps and foreign materials are unavoidable [13-15]. On the other hand, indium tin oxide (ITO) has been widely used in GaN-based LEDs as transparent conductive layer (TCL) to improve current spreading in $\mathrm{p}-\mathrm{GaN}$ layer. Popular-use thickness of ITO on $\mathrm{p}-\mathrm{GaN}$ is about $200-245 \mathrm{~nm}$. However, the thickness is too thin to etch due to influence of the current spreading of GaN-based LEDs. In this paper, we proposed the highly thick ITO as the TCL of GaN LEDs. Because both LED operation voltage and series resistance could be reduced due to the reduction in current crowding, on the other hand, the thickness provides the enough depth for the roughening surface on ITO without any damages in electrical properties. In order to produce nanoscale patterns on the surface of GaN-based LEDs, so far, few researches report chemical and physical deposition techniques on the fabricating of $\mathrm{ZnO}$ nanoparticles. As our previous report [16], SILAR-deposition $\mathrm{ZnO}$ nanoparticles were used to produce the $\mathrm{SiN}_{x}$ nanopillars on the surface of GaN-based LEDs. The SILAR method approaches based on the soft chemical technique have attracted increasing attention [17], due to its high reliability, low cost, and largearea deposition compared with other methods. In this paper, high-thickness ITO was used as the transparent contact 
layer of GaN LEDs, and different densities of self-assembly $\mathrm{ZnO}$ nanomasks and etching time were used to produce the roughened surface including the ITO surface, p-GaN layer around the edge of the mesa, the sidewall, and the $n-G a N$ layer on GaN-based LEDs. In addition, the resulting light output power efficiency of LEDs with roughness surfaces is significantly higher than that of a conventional LED.

\section{Experimental}

The GaN-based LED was grown on $c$-axis sapphire substrates by using low-pressure metal-organic chemical-vapor deposition. N-type GaN epitaxial layers, which included a $1 \mu \mathrm{m}$ thick undoped GaN layer and a $2 \mu \mathrm{m}$ thick Si-doped n-GaN layer, were fabricated on sapphire substrates as templates for the subsequent regrowth process, before the growth of LED structures. The five periods InGaN/GaN multiple quantum well (MQW) with emission wavelength in the blue region and a $150 \mathrm{~nm}$ p-GaN layer were fabricated. The LEDs had a mesa structure with an area of $300 \times 300 \mu \mathrm{m}^{2}$. Before fabricating the LEDs using $\mathrm{SiO}_{2}$, a $1 \mu \mathrm{m}$ thick ITO film was deposited on the top of GaN-based LEDs by radio frequency magnetron sputtering, and we also prepared a $200 \mathrm{~nm}$ thickness of ITO film LED to be compared. $\mathrm{Cr} / \mathrm{Au}$ layers as the $\mathrm{p}$ - and ncontact electrodes were fabricated. Figure 2 demonstrates the schematic diagram of the pattern transfer procedure. A $300 \mathrm{~nm}$ thick $\mathrm{SiO}_{2}$ film was deposited on an ITO layer by PECVD as Figure 2(b). The metal contacts were covered with a photoresist layer by photolithography in order to prevent the $\mathrm{SiO}_{2}$ which was grown on the ohmic contact electrodes. In Figure 2(c), the $\mathrm{ZnO}$ nanoparticles were grown on the top of $\mathrm{SiO}_{2}$ film as the etching mask by SILAR. The detailed procedures of $\mathrm{ZnO}$ nanoparticles in one cycle are shown in Figure 1 and described in our previous report [16]. In this case, the samples were dipping in $95^{\circ} \mathrm{C}$ DI water: ethylene glycol $=1: 1$ for $20 \mathrm{~s}$ to form $\mathrm{ZnO}$ nanoparticles on $\mathrm{SiO}_{2}$ film. Furthermore, we etched $\mathrm{SiO}_{2}$ by using ICP to get the $\mathrm{SiO}_{2}$ nanopillars on all the surfaces using a $\mathrm{CF}_{4}$ gas in the first case as shown in Figure 2(e). We removed the $\mathrm{ZnO}$ nanoparticles and photoresist by HCL and acetone. As shown in Figure 2(f), the second case, after etching $\mathrm{SiO}_{2}$, we intentionally fabricated nanostructures on both ITO and GaN simultaneously using a $\mathrm{Cl}_{2}-\mathrm{Ar}$ gas mixture. In the third case, after the second case, we removed the $\mathrm{SiO}_{2}$ nanoparticles and photoresist by BOE and acetone as shown in Figure 2(g). The characteristics of current-voltage $(I-V)$ and current-power were measured at room temperature using Keithley 2430 source meter combined with an integrating sphere and a spectrum meter.

\section{Results and Discussion}

Figure 3 shows the four cases of the $30^{\circ}$ tile-view SEM images of the surface on GaN-based LEDs at ratio of DI water: ethylene glycol = 1:1; (a) conventional LED I (with $240 \mathrm{~nm}$ thick ITO) and LED II (with $1000 \mathrm{~nm}$ thick ITO); (b) LED III (with $1000 \mathrm{~nm}$ thick ITO) with $\mathrm{SiO}_{2}$ nanopillars; (c)
LED IV (with $1000 \mathrm{~nm}$ thick ITO) with $\mathrm{SiO}_{2} / \mathrm{ITO}, \mathrm{SiO}_{2} / \mathrm{p}$ $\mathrm{GaN}$, and $\mathrm{SiO}_{2} / \mathrm{n}-\mathrm{GaN}$ nanopillars; (d) LED V (with $1000 \mathrm{~nm}$ thick ITO) with ITO, $\mathrm{p}-\mathrm{GaN}$, and $\mathrm{n}-\mathrm{GaN}$ by removing $\mathrm{SiO}_{2}$. Figure 3(a) shows the first case, LED I and LED II, and Figures 3(a1) and 3(a2) display the magnified images of the flat surface ITO, $\mathrm{p}-\mathrm{GaN}$, and $\mathrm{n}-\mathrm{GaN}$ regions, respectively. After the $\mathrm{ZnO}$ nanoparticles were deposited at $95^{\circ} \mathrm{C}$ DI water : ethylene glycol = 1: 1 and etching, Figure 3(b) (LED II) shows that the diameter and height of $\mathrm{SiO}_{2}$ nanopillars were from 100 to $450 \mathrm{~nm}$ and $300 \mathrm{~nm}$, respectively, and the spacing was approximately $1 \mu \mathrm{m}$. Figures 3(b1) and 3(b2) show the magnified images of $\mathrm{SiO}_{2}$ nanopillars on the surfaces of ITO, $\mathrm{p}-\mathrm{GaN}$, and $\mathrm{n}-\mathrm{GaN}$, respectively. Subsequently, we intentionally fabricated nanostructures on both ITO and GaN simultaneously using a $\mathrm{Cl}_{2}$-Ar gas mixture and Figure 3(c) (LED III) shows that diameter and height of nanopillars were from 100 to $350 \mathrm{~nm}$ and $250 \mathrm{~nm}$, respectively. The spacing was also approximately $800 \mu \mathrm{m}$. Figures 3(c1) and 3(c2) display the magnified images of $\mathrm{SiO}_{2} / \mathrm{ITO}, \mathrm{SiO}_{2} / \mathrm{p}-\mathrm{GaN}$, and $\mathrm{SiO}_{2} / \mathrm{n}$ GaN nanopillars on surface, respectively. Furthermore, we removed the $\mathrm{SiO}_{2}$ by BOE from top of surface of $\mathrm{GaN}$-based LED which was shown in Figure 3(d) (LED IV). Figures 3(c1) and 3(c2) display the magnified images of the ITO, p-GaN, and $\mathrm{n}-\mathrm{GaN}$ nanopillars on surface, respectively. The average depth of the ITO pattern is around $200 \mathrm{~nm}$. We can figure out from Figures 3(cl) and 3(d1) that after removing $\mathrm{SiO}_{2}$, the shape of patterns would become ladder-shaped and we will analyze the effect of this situation in detail later.

Figure 4(a) shows the current-voltage $(I-V)$ characteristics of thinness of conventional LED and thickness of ITO of conventional LED with and without nanostructures. At a current of $20 \mathrm{~mA}$ injection, it was found that forward voltages were $3.5,3.22,3.28,3.27$, and $3.27 \mathrm{~V}$ for LED I, LED II, LED III, LED IV, and LED V, respectively. It showed that both of the forward voltages and series resistance of thickness of ITO of conventional LED are lower than thinness of conventional LED because much more effectiveness for the current crowding reduction is increasing the electron concentration in the contact layer of the LED, and it also found that the $I-V$ characteristics of GaN-based LEDs with and without nanopillars were very similar. The slightly higher forward voltages could result from that lone time dry etching damage to ITO film, which affects the sheet resistance [18] and current spreading and even GaN film. Figure 4(b) demonstrates light output power of thinness of conventional LED and thickness of ITO of conventional LED with and without nanopillars at $20 \mathrm{~mA}$ driving current. The output intensities of these LEDs increased with injection current when the injection current was small and can be seen. Furthermore, it was found that output powers observed from the three cases of LEDs with nanopillars were all larger than that observed from the conventional LED again. With $20 \mathrm{~mA}$ injection current, it was found that output powers of these LEDs were 6.61, 6.48, 6.96, 7.73, and $7.12 \mathrm{~mW}$ for the LED I, LED II, LED III, LED IV, and LED V, respectively. It showed that light output power of thickness of ITO of conventional LED is lower than thinness of conventional LED about $2 \%$ due to free carrier absorption of emitted light in the ITO film. It was also shown that smaller output power of LED I and LED II is attributed to Fresnel 


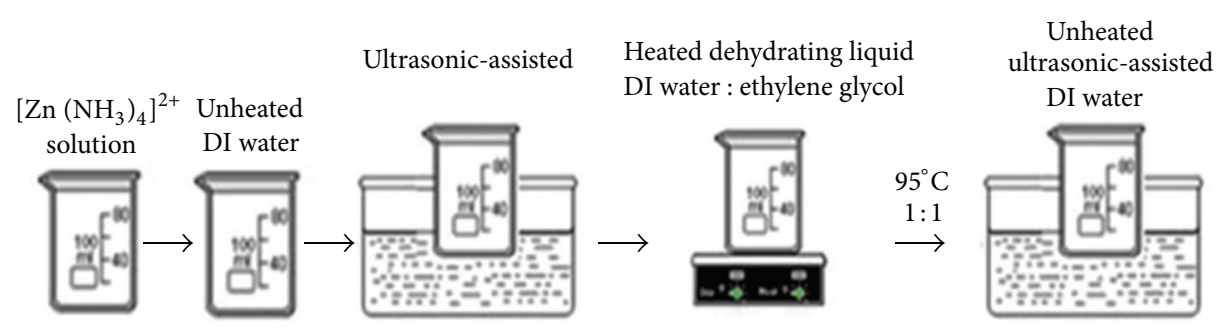

FIGURE 1: Process schemes of the various rinsing procedures.

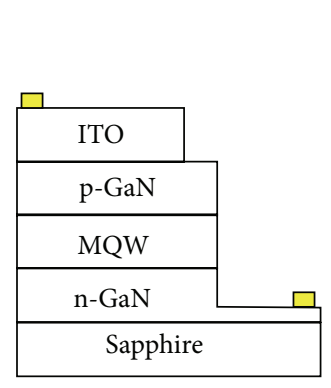

(a)

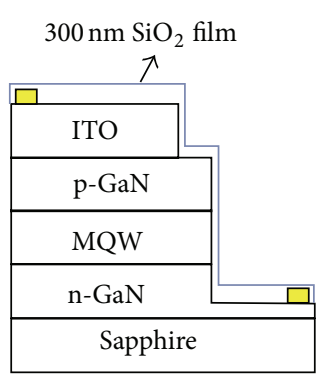

(b)

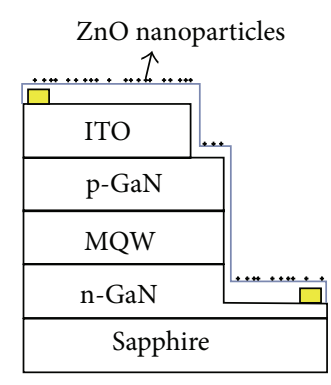

(c)

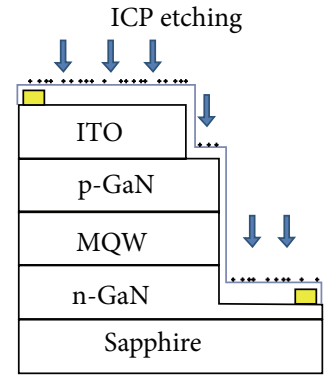

(d)

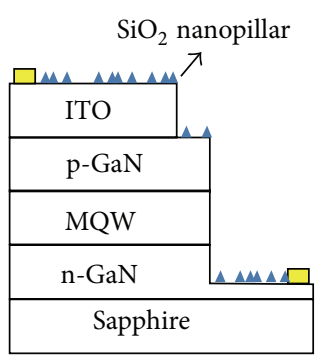

(e)

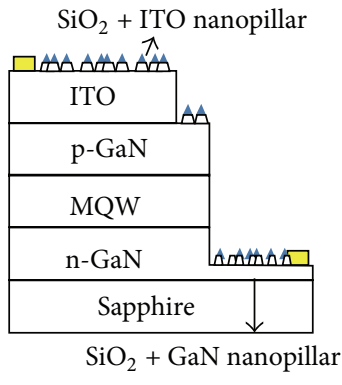

(f)

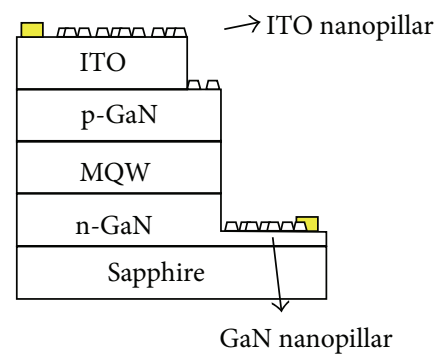

(g)

FIGURE 2: Schematic diagram of the pattern transfer procedure of nanostructure LED: (a) conventional LED (LED-II); (b) deposited $300 \mathrm{~nm}$ $\mathrm{SiO}_{2}$ on the surface by PECVD; (c) deposition of $\mathrm{ZnO}$ nanoparticles on the surface by SILAR; (d) ICP etching; (e) LED with SiO ${ }_{2}$ nanopillars (LED-III); (f) LED with $\mathrm{SiO}_{2}$, ITO, and GaN nanopillars (LED-IV); (g) LED with ITO and GaN nanostructure (LED-V).

reflection. In other words, we can enhance the light output power at $20 \mathrm{~mA}$ by $7 \%, 20.3 \%$, and $9.1 \%$ with nanostructures for LED III-LED V, respectively, compared with LED II. The increase of light output power of nanostructure suggests that the reduction of Fresnel reflection on the surface is a major cause of an increased light-extraction efficiency. It also found that the light-extraction efficiency was increased following the higher density of nanostructure. It is attributed to more nanopillars increasing cause of higher light scattering effect by the nanopillars on the ITO and GaN surfaces.

Figure 5 shows the schematic cross-section diagrams of the LEDs in conventional LED and LED with nanopillars, respectively. As Figure 5(a), most of the light generated in the MQWs active layers is trapped inside the device because of the refractive index difference between the semiconductor and the surrounding medium. For nano tructures LEDs, the surfaces of ITO, p-GaN, and n-GaN were all nanopatterned. The trapped guided modes could multiply scattering and find more chances for radiation out from the device and they suggest that the reduction of Fresnel reflection on the surface is a major cause of increased light-extraction efficiency.

Figure 6 shows the schematic cross-section diagrams of the LEDs. In Figure 6(a), it shows that most of the light generated in the MQWs active layer is trapped inside the device due to the refractive index difference between the semiconductor and the surrounding medium. The $\mathrm{SiO}_{2}$ nanopillars are useful for light extraction, since more surfaces of the nanopillars can provide more opportunities for light escaping and reduce Fresnel refraction because $\mathrm{SiO}_{2}$ refractive index was lower than ITO as shown in Figure 6(b), but partial plane ITO of surface can cause Fresnel refraction. In Figure 6(c), it shows more enhancing of light output power by further etching of ITO because ITO became rough resulting in reducing Fresnel refraction. Furthermore, we removed the $\mathrm{SiO}_{2}$ nanopillars by $\mathrm{BOE}$ and got the ladder-shaped ITO patterned. It was found that light output power reduced because the surface of the ladder-shaped ITO was plane and caused the Fresnel reflection again. 


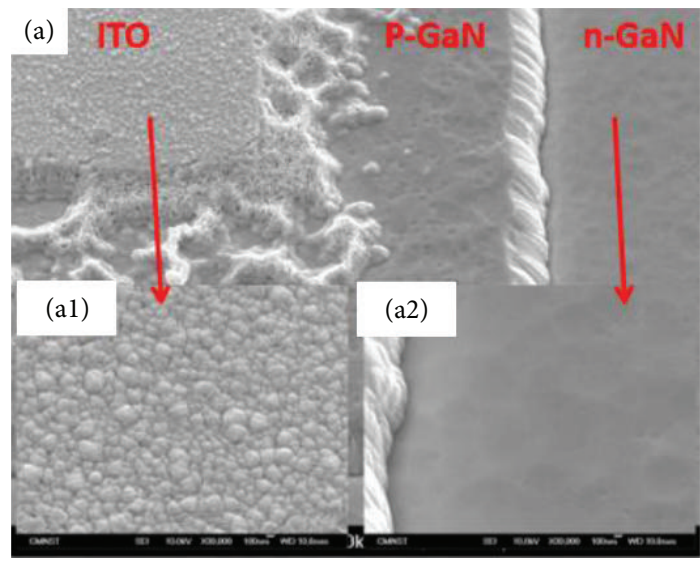

(a)

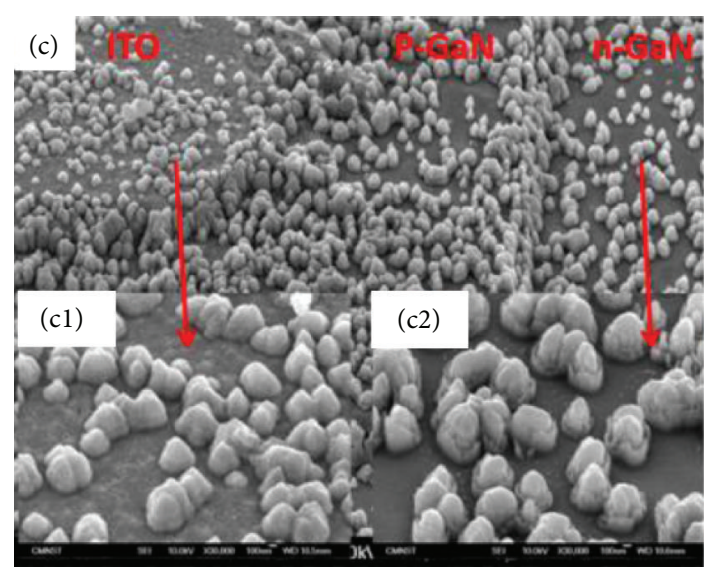

(c)

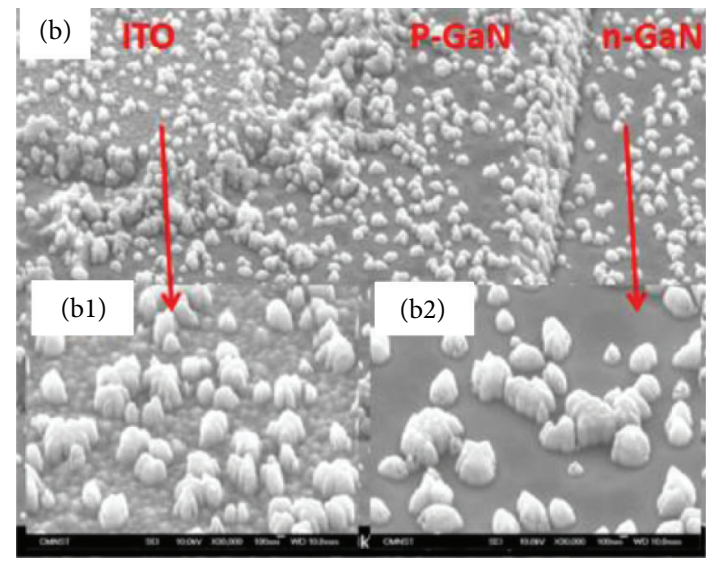

(b)

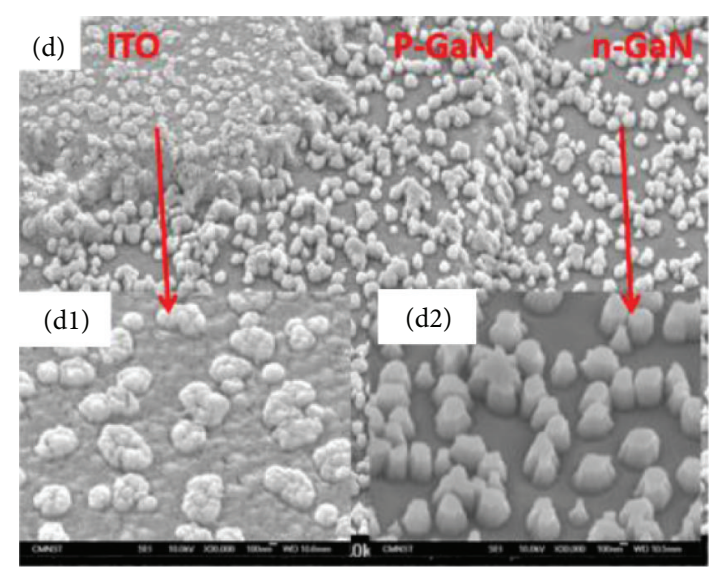

(d)

FIGURE 3: SEM images of the surface: (a) flat surface: LED I and LED II without nanopatterns, (b) LED III with SiO ${ }_{2}$ nanopillars, (c) LED IV with $\mathrm{SiO}_{2} / \mathrm{ITO}, \mathrm{SiO}_{2} / \mathrm{p}-\mathrm{GaN}$, and $\mathrm{SiO}_{2} / \mathrm{n}-\mathrm{GaN}$ nanopillars, and (d) LED V with ITO, p-GaN, and n-GaN nanopillars by removing SiO .

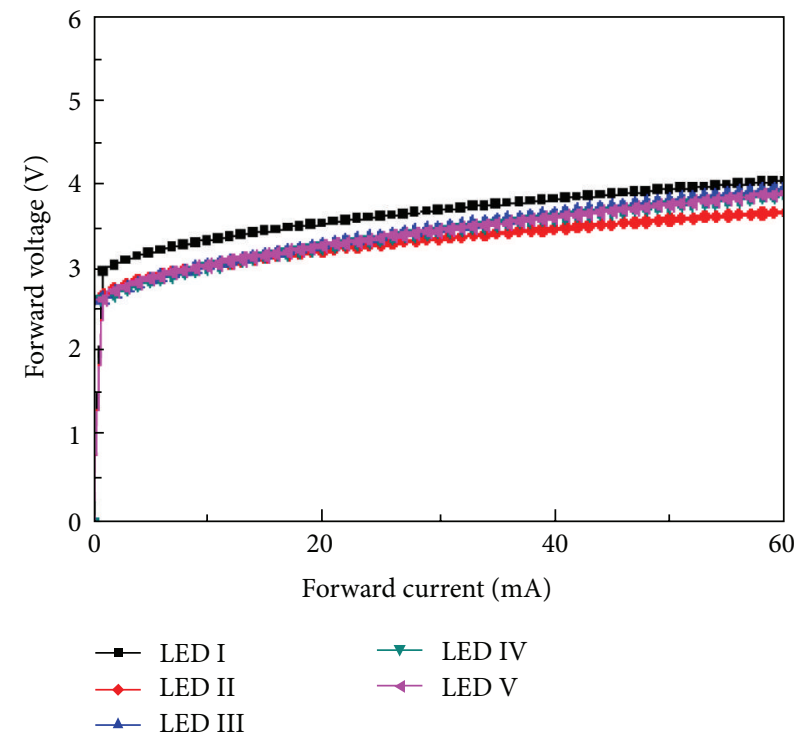

(a)

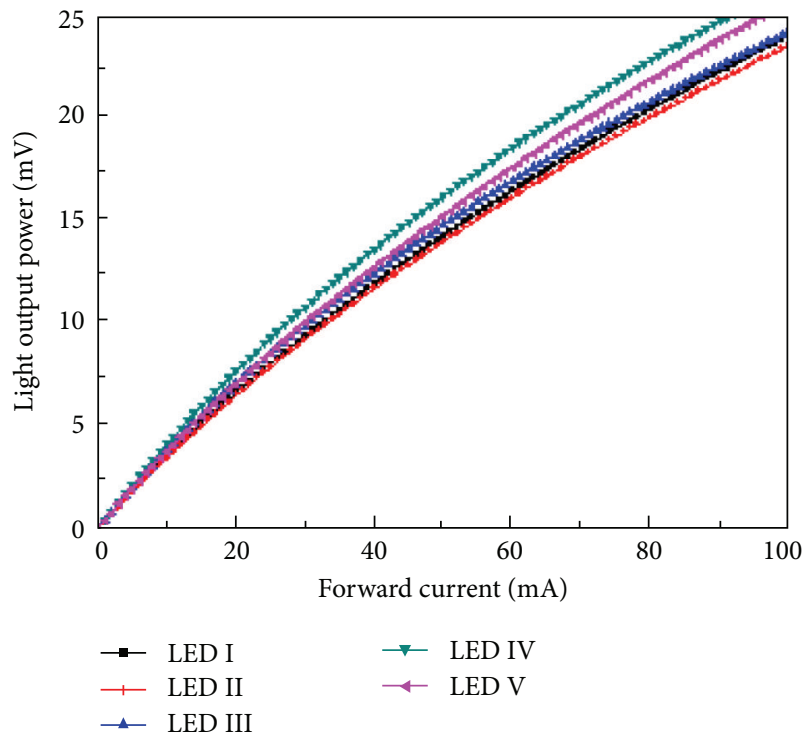

(b)

FIgURE 4: (a) I- $V$ curves of GaN-based LEDs with and without nanopillar $\mathrm{SiO}_{2}$. (b) Light output current characteristics of GaN-based LEDs with and without nanopillars. 


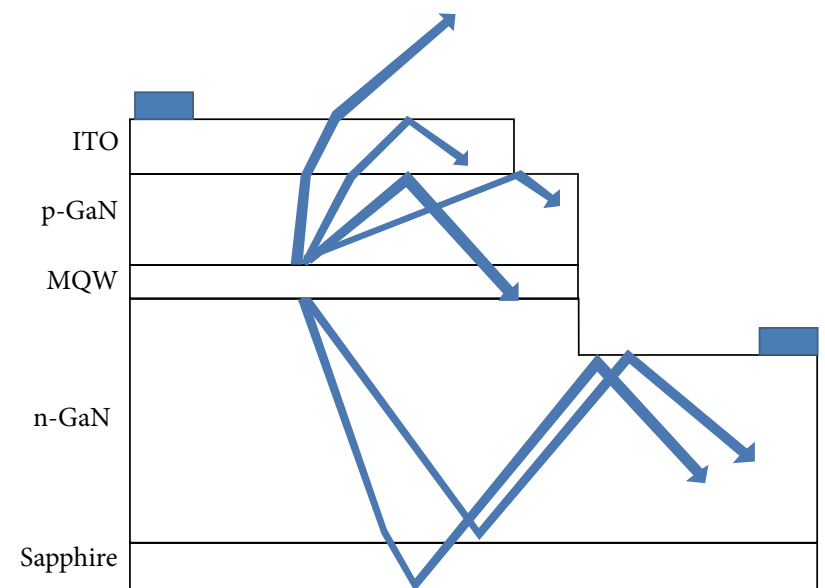

(a)

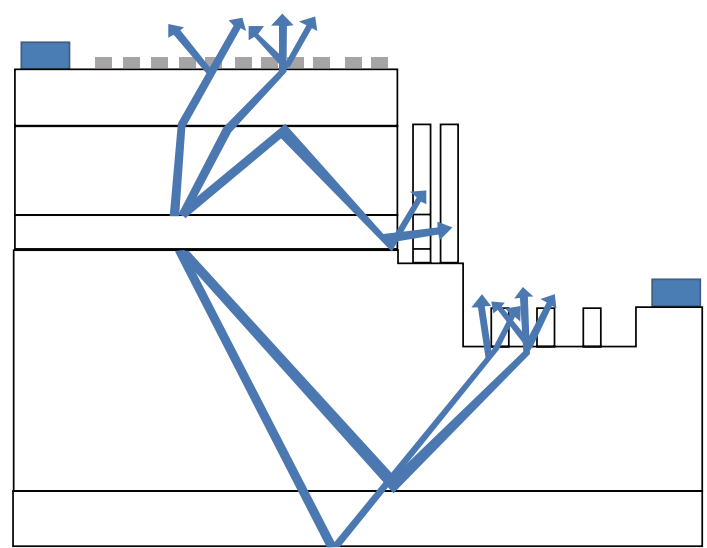

(b)

FIGURE 5: Schematic diagrams of (a) conventional GaN-based LED and (b) GaN-based LED with nanopillars.

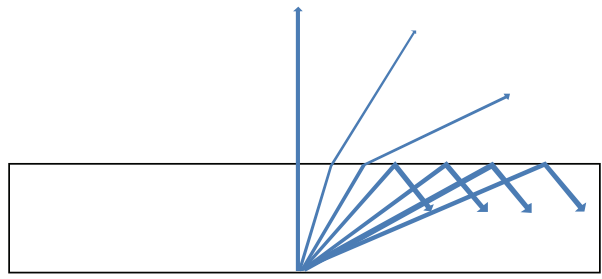

(a)

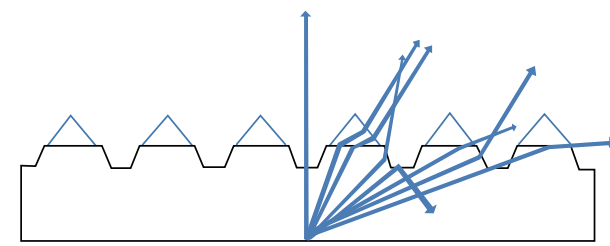

(c)

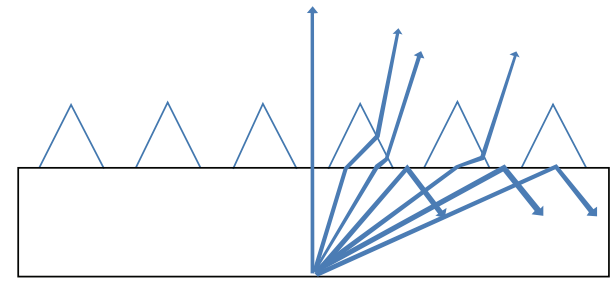

(b)

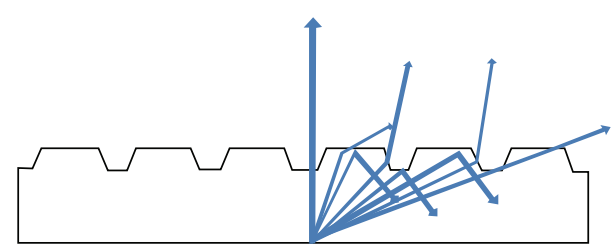

(d)

FIgURE 6: Schematic diagrams of the difference of material and shape of nanostructures: (a) LED with flat surface ITO, (b) LED with SiO 2 nanopillars, (c) LED with $\mathrm{SiO}_{2} /$ ITO nanopillars, and (d) LED with ITO nanopillars.

\section{Conclusion}

In summary, it was found that thick ITO as TCL can reduce current crowding and series resistance. By using $\mathrm{ZnO}$ nanoparticles as dry etching nanomasks on $\mathrm{SiO}_{2}$ film before $\mathrm{SiO}_{2}$ etching by ICP, and then further etching ITO and GaN. In addition, we have successfully demonstrated a feasible method to enhance light extraction by producing nanostructures on overall GaN-based LED surfaces by lowtemperature SILAR method. The optimal enhancement of light output power of the GaN-based LEDs with nanostructures is achieved up to $20.3 \%$ compared to that of the conditional LED at injection current of $20 \mathrm{~mA}$. The enhancement is attributed to the reduction of Fresnel reflection and scattering effects by nanopillars on the whole surface of the LED.

\section{Acknowledgment}

The authors would like to thank the National Science Council of Taiwan, Taiwan, under Contract nos. NSC 101-2262-E-024002-CC3 and NSC 101-2221-E-024-003-MY3.

\section{References}

[1] T. Mukai, M. Yamada, and S. Nakamura, "Characteristics of InGaN-based UV/blue/green/amber/red light-emitting diodes," Japanese Journal of Applied Physics, vol. 38, no. 7, pp. 39763981, 1999.

[2] T. Fujii, Y. Gao, R. Sharma et al., "Use of patterned laser liftoff process and electroplating nickel layer for the fabrication of vertical-structured GaN-based light-emitting diodes," Applied Physics Letters, vol. 84, pp. 855-857, 2004.

[3] D. W. Kim, H. Y. Lee, M. C. Yoo, and G. Y. Yeom, "Highly efficient vertical laser-liftoff GaN-based light-emitting diodes formed by optimization of the cathode structure," Applied Physics Letters, vol. 86, no. 5, Article ID 052108, 3 pages, 2005.

[4] H.-W. Huang, C. C. Kao, J. T. Chu, H. C. Kuo, S. C. Wang, and C. C. Yu, "Improvement of InGaN-GaN light-emitting diode performance with a nano-roughened $\mathrm{p}-\mathrm{GaN}$ surface," IEEE Photonics Technology Letters, vol. 17, no. 5, pp. 983-985, 2005.

[5] H.-W. Huang, J. T. Chu, C. C. Kao et al., "Enhanced light output of an InGaN/GaN light emitting diode with a nano-roughened 
p-GaN surface," Nanotechnology, vol. 16, no. 9, pp. 1844-1848, 2005.

[6] C.-C. Kao, H.-C. Kuo, H.-W. Huang et al., "Light-output enhancement in a nitride-based light-emitting diode with 22oundercut sidewalls," IEEE Photonics Technology Letters, vol. 17, no. 1, pp. 19-21, 2005.

[7] Y. J. Lee, J. M. Hwang, T. C. Hsu et al., "Enhancing the output power of GaN-based LEDs grown on wet-etched patterned sapphire substrates," IEEE Photonics Technology Letters, vol. 18, no. 10, pp. 1152-1154, 2006.

[8] J. H. Lee, J. T. Oh, Y. C. Kim et al., "Enhanced output power of InGaN-based light-emitting diodes with AlGaN/GaN twodimensional electron gas structure," IEEE Photonics, vol. 20, no. 18, pp. 1563-1565, 2008.

[9] C. H. Chiu, H. C. Kuo, C. E. Lee et al., "Fabrication and characteristics of thin-film InGaN-GaN light-emitting diodes with $\mathrm{TiO}_{2} / \mathrm{SiO}_{2}$ omnidirectional reflectors," Semiconductor Science and Technology, vol. 22, no. 7, pp. 831-835, 2007.

[10] S. C. Hung, Y. K. Su, S. J. Chang et al., "Self-formation of GaN hollow nanocolumns by inductively coupled plasma etching," Applied Physics A, vol. 80, no. 8, pp. 1607-1610, 2005.

[11] S.-C. Hung, Y.-K. Su, S.-J. Chang, L.-W. Ji, D. Shen, and C. H. Huang, "InGaN/GaN MQD p-n junction photodiodes," Physica E, vol. 30, no. 1-2, pp. 13-16, 2005.

[12] Y. K. Su, S. J. Chang, L. W. Ji et al., "InGaN/GaN blue light-emitting diodes with self-assembled quantum dots," Semiconductor Science and Technology, vol. 19, no. 3, pp. 389-392, 2004.

[13] C. H. Chiu, C. E. Lee, C. L. Chao et al., "Enhancement of light output intensity by integrating $\mathrm{ZnO}$ nanorod arrays on GaN-based LLO vertical LEDs," Electrochemical and Solid-State Letters, vol. 11, no. 4, pp. H84-H87, 2008.

[14] J. Zhong, H. Chen, G. Saraf et al., "Integrated $\mathrm{ZnO}$ nanotips on GaN light emitting diodes for enhanced emission efficiency," Applied Physics Letters, vol. 90, no. 20, Article ID 203515, 2007.

[15] R.-H. Horng, S.-H. Huang, C.-C. Yang, and D.-S. Wuu, "Efficiency improvement of GaN-based LEDs with ITO texturing window layers using natural lithography," IEEE Journal on Selected Topics in Quantum Electronics, vol. 12, no. 6, pp. 11961201, 2006.

[16] S.-C. Shei, S.-J. Chang, and P.-Y. Lee, "Rinsing effects on successive ionic layer adsorption and reaction method for deposition of $\mathrm{ZnO}$ thin films," Journal of the Electrochemical Society, vol. 158, no. 3, pp. H208-H213, 2011.

[17] T. P. Niesen and M. R. De Guire, "Review: deposition of ceramic thin films at low temperatures from aqueous solutions," Solid State Ionics, vol. 151, no. 1-4, pp. 61-68, 2002.

[18] X.-D. Gao, X.-M. Li, W.-D. Yu, L. Li, J.-J. Qiu, and F. Peng, "Low-temperature deposition of transparent $\mathrm{ZnO}$ films by the ultrasonic-mediated stepwise method," Solar Energy Materials and Solar Cells, vol. 91, no. 6, pp. 467-473, 2007. 

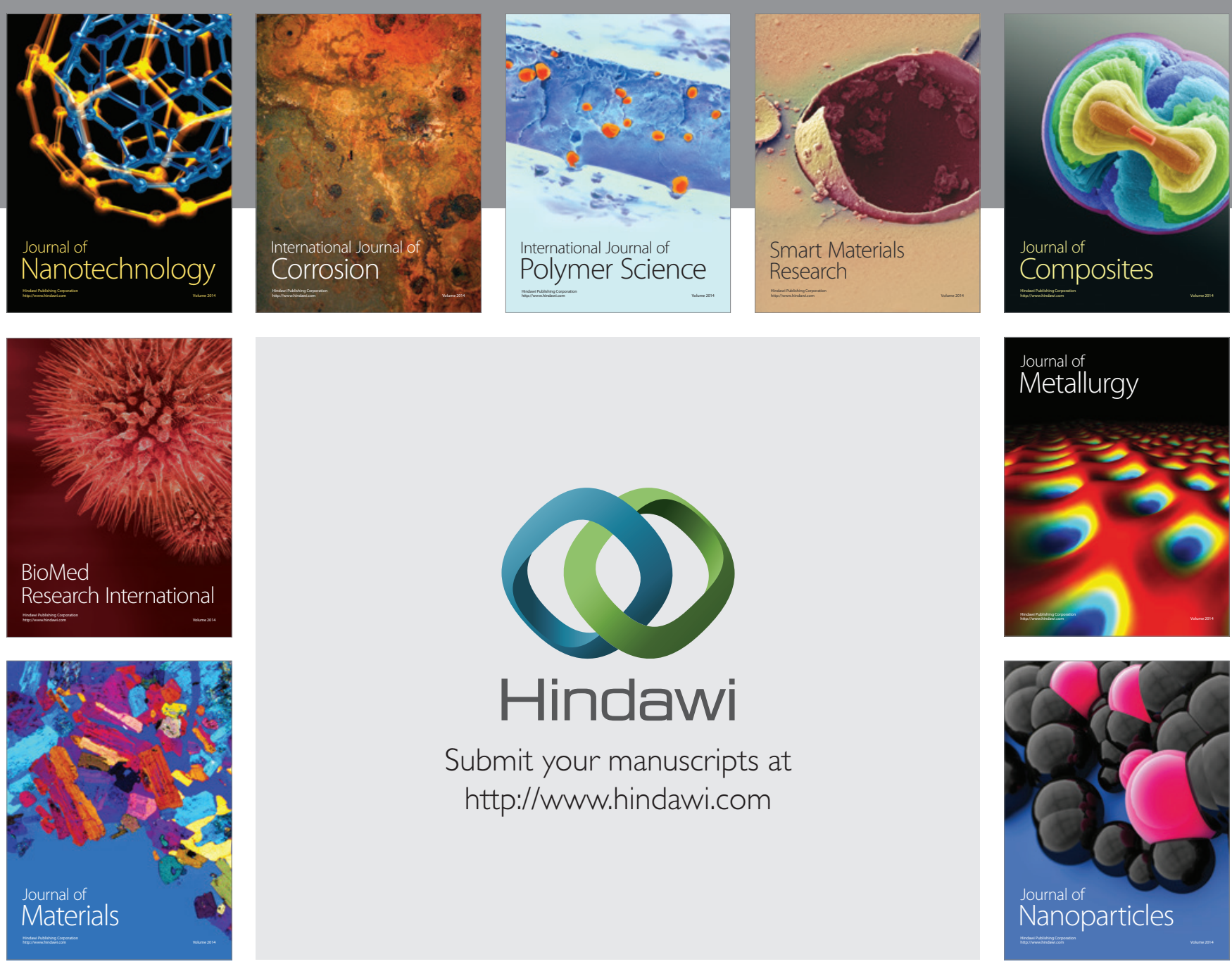

Submit your manuscripts at http://www.hindawi.com
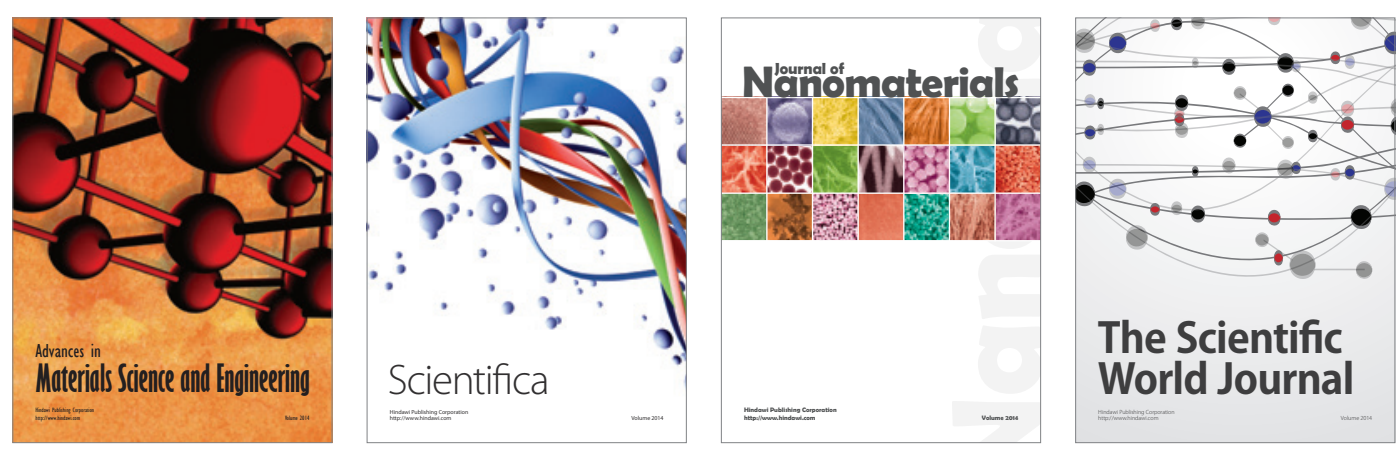

\section{The Scientific World Journal}
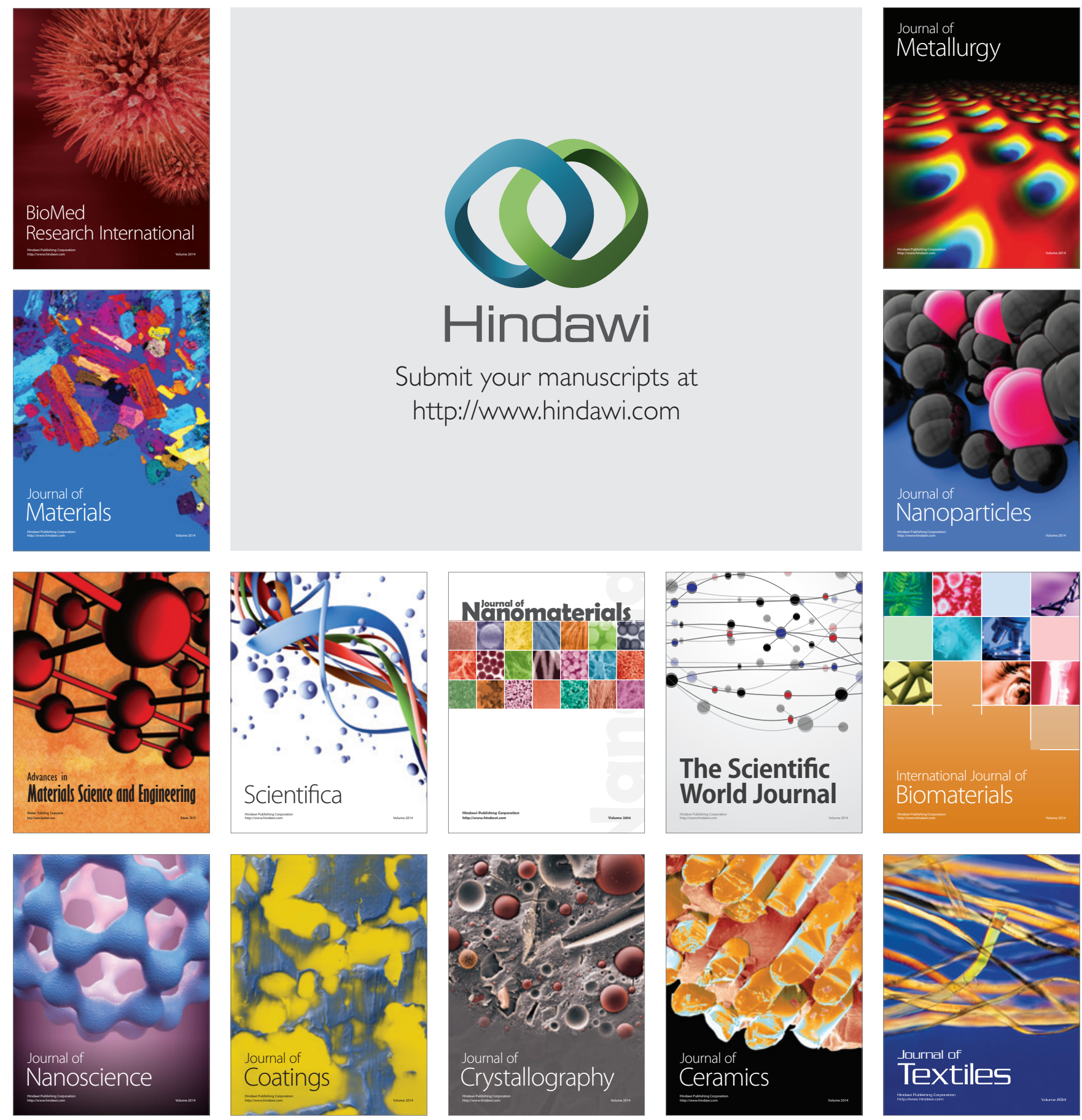